\title{
Atypical Presentation of Laryngeal Histoplasmosis
}

\author{
M.B. Bharathi, Enu Malik, A.R. Babu \\ Department of Otolaryngology, JSS Medical College and Hospital, Mysore, Karnataka, India.
}

\section{Corresponding Author: \\ Dr. Enu Malik \\ Email: enumkukreja@gmail.com}

This is an Open Access article distributed under the terms of the Creative Commons Attribution License (creativecommons.org/ licenses/by/3.0).

Received : September 8, 2017

Accepted : December 23, 2017

Published : January 15, 2018

\begin{abstract}
Background: Medical literature has documented less than 100 cases of histoplasmosis till date. It is an endemic granulomatous fungus disease, which rarely infects the larynx in immunologically competent patients. Case Report: A 68 year old male presented with difficulty in swallowing and progressive change in voice for one month which clinically suggested laryngeal tuberculosis. Laryngoscopy revealed edematous epiglottis and aryepiglottic fold. Histopathological biopsy illustrated sheets of histiocytes with numerous forms of Histoplasma capsulatum. Conclusion: Laryngeal histoplasmosis should be considered in the differential diagnosis of a patient with hoarseness of voice and moreover, in the differential diagnosis of tumors in the vocal folds, causing hoarseness.
\end{abstract}

Keywords: Histoplasma, Hoarseness, Laryngeal Tuberculosis, Laryngoscopy, Vocal Cords.

\section{Introduction}

Recently, granulomatous diseases caused by infectious agents have occurred more frequently than they were described before. Such occurrence may be explained by the increase in number of immunocompromised patients or patients treated by immunosuppressive agents. Laryngeal histoplasmosis is a rare condition that may mimic tuberculosis or even laryngeal cancer and must be considered in the differential diagnosis of a patient with hoarseness of voice and thus of the tumors in the vocal folds resulting in hoarseness [1]. Amphotericin B is the drug of choice for the treatment of this disease, although other drugs have also been used.

It is a granulomatous disease of worldwide distribution caused by a dimorphic fungus Histoplasma capsulatum (H. capsulatum). $H$. capsulatum is acquired by inhalation of mycelial fragments of the fungus. Histoplasmosis is less frequently reported in Asia and Europe. We report an unusual case of histoplasmosis presenting as a laryngeal epiglottitis in an immunocompetent person.

\section{Case Report}

A 68 year old male presented with difficulty in swallowing which was insidious in onset and gradually progressive from past one month and was more to solids than liquids. It was also associated with burning sensation in throat. He also had associated change in voice which was insidious in onset with no noticeable fatigability.

Patient was a known case of bronchial asthma which was under control with routine bronchodilator therapy only (no history of use of steroids hence ruling out immunocompromised status) and hypertension for which he was on regular treatment. He also had history of cardiac bypass surgery four years ago and he was on a daily aspirin dose. Patient was also an alcoholic (upto $180 \mathrm{~mL} /$ day) and used to smoke 2 packs/ day for 40 years. There were no lesions observed on the skin. The general physical examination was normal. Flexible laryngoscopic examination showed edematous epiglottis and aryepiglottic folds with multiple ulcerative lesions involving posterior pharyngeal wall, arytenoids and aryepiglottic folds. The mobility of bilateral vocal cords was normal. 
Tubercular laryngitis was suspected. Biopsy was taken and histopathological examination showed stratified squamous epithelium and sub-epithelium displaying sheets of histiocytes and numerous forms of Histoplasma capsulatum. Tests for human immunodeficiency virus and hepatitis B antigen were negative. Blood urea and creatinine were found to be 66 (normal level: 10-40 mg/dL) and $1.7(0.5-1.4 \mathrm{mg} / \mathrm{dL})$ respectively. Total proteins and albumin were comparatively decreased [5.46 gm/dL (normal level: 6.4-8.3 g/dL) and $2.80 \mathrm{gm} / \mathrm{dL}$ (normal level: $3.5-5 \mathrm{~g} / \mathrm{dL}$ ) respectively]. On the other hand serum glutamic oxaloacetic transaminase [61 (10-40 units/L)], serum glutamic pyruvic transaminase [45 (10-40 units/L)], alkaline phosphatase [2126 (40-145 units/L)], gamma glutamyltransferase $[328$ (9-48 units/L)] were raised. Ultrasound abdomen showed massive hepato-splenomegaly with minimal ascites, these were coincidental associated findings which appear to be the part of alcoholic liver disease as a separate symptom complex.

2D ECHO was done and showed ischemic heart disease, coronary artery bypass grafting and mitral valve repair, mild pulmonary hypertension and ejection fraction was noted to be $45 \%$. The patient was started on itraconazole $200 \mathrm{mg}$ thrice daily for five days and then tapered to $200 \mathrm{mg}$ once a day following which patient's symptoms improved. $10^{\text {th }}$ day flexible laryngoscopy revealed decrease in edema and lesions were reduced in size. Thus, the treatment was continued for another three months. Repeat laryngoscopic examination showed healed granulations over epiglottis and aryepiglottic folds.

\section{Discussion}

Histoplasmosis is a granulomatous disease of universal distribution caused by a dimorphic intracellular fungus, Histoplasma capsulatum. According to Goodwin Jr. et al. [2], it was Monbreun, in 1934, who first saw that the Histoplasma grows as a filamentous fungi and also as a yeast. It also

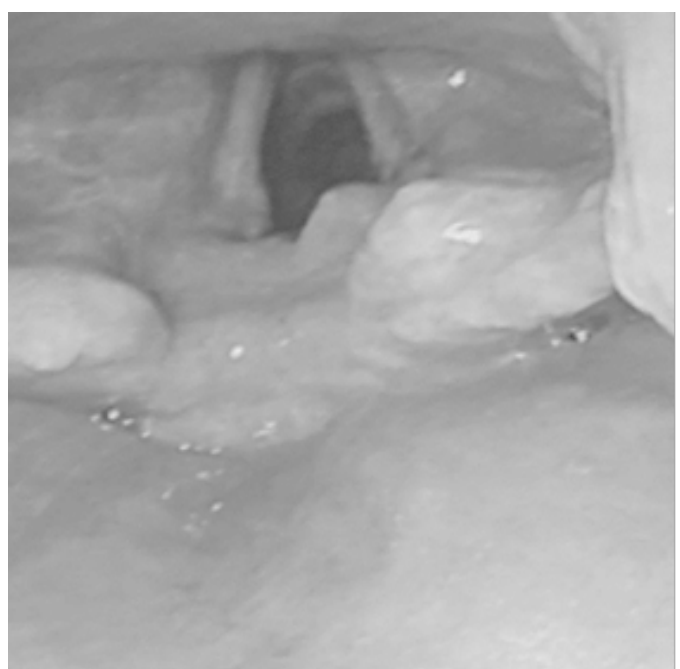

Fig.1: Endoscopic image of the patient after successful treatment.

affects thereticular-endothelial system. This fungus can be found in caves or hen houses. Various factors such as amount of organisms inhaled or immunity effects its progression.

Acute presentation in children or in severely immunosuppressed patients may present with fever, weight loss, hepato-splenomegaly, shock and disseminated intra-vascular coagulation. In the chronic forms fever, weight loss,asthenia and oral mucosa lesions are very common. Chronic pulmonary histoplasmosis is very much similar to tuberculosis, with slow progressive fever, weight loss and respiratory symptoms with chest X-rays showing areas of excessive cavitation and fibrosis. It must especially be considered in patients who do not respond to tuberculosis treatment.

For most of the immunocompetent patients, it is asymptomatic or symptoms are suggestive of flu [3]. Typical initial manifestations of laryngeal histoplasmosis include hoarseness, dysphagia and odynophagia as well as fatigue and weight loss [4]. Staloff et al. [5] commented that the spread is very rare, as a clinically apparent infection, happening more frequently in immunosuppressed and elderly patients. The areas commonly involved are: bone marrow, lymph nodes, adrenal glands 
and gastrointestinal tract, just as the tongue and oral mucosa.

Histoplasmosis may occur in three forms: (i) primary acute pulmonary form, (ii) chronic pulmonary and (iii) disseminated form. The mucosal involvement could be oropharyngeal or laryngeal involvement. The incidence of oral manifestation is $25-45 \%$ in the disseminated form of the disease. Laryngeal involvement is usually associated with dissemination [5]. In our patient, there were however no systemic symptoms like fever, weight loss or evidence of adrenal gland involvement.

The oral cavity was not involved and there was no mucocutaneous involvement/rash. The patient however had hepato-splenomagly which was probably related to his other comorbidity of alcoholic liver disease. Thus, it can be concluded that this was a case of purely a laryngeal affection of histoplasmosis without any systemic involvement (abdominal, cutaneous or others) and also the presentation was atypical (edematous, non ulcerative, lesion of the submucosal layer of the epiglottis with loss of surrounding folds).

\section{Conclusion}

A high index of suspicion is needed to make a diagnosis of histoplasmosis early especially in immunocompetent host residing in non-endemic areas. Clinical features and laboratory features are identical to the commonly seen granulomatous infections such as disseminated tuberculosis. It also mimics laryngeal carcinoma. Our patient presented with laryngeal epiglottitis and only a biopsy of the lesion helped us establish the diagnosis. Diagnosis needs to be confirmed via smears or cultures of tissues obtained from these affected sites as early diagnosis and antifungal treatment results in remission or cure, usually with no sequelae.

Contributors: MBB: manuscript editing, critical updates; EM: manuscript writing, literature review; ARB: critical inputs into the manuscript and literature review. EM will act as guarantor. All authors approved the final version of this manuscript.

Funding: None; Competing interests: None stated.

\section{References}

1. Subramaniam S, Abdullah AH, Hairuzah I. Histoplasmosis of larynx. Med J Malaysia. 2005;60:386-388.

2. Goodwin RA Jr, Shapiro JL, Thurman GH, Thurman SS, Des Prez RM. Disseminated histoplasmosis: Clinical and pathologic correlations. Medicine (Baltimore). 1980;59:1-33.

3. Wolf J, Blumberg HM, Leonard MK. Laryngeal histoplasmosis. Am J Med Science. 2004;327:160-162.

4. Reibel JF, Jarhsdorfer RA, Johns MM, Cantrell RW. Histoplasmosis larynx. Otolaryngology Head Neck Surg. 1982;90:740-743.

5. Staloff RT, Wilborn A, Prestipino A, Hawkshaw M, Heuer RJ, Cohn J. Histoplasmosis of the larynx. Am J Otolaryngology. 1993;14:199-205. 